\title{
Adapting Personas for Use in Security Visualization Design
}

\author{
J. Stoll, D. McColgin, M. Gregory, V. Crow, and W.K. Edwards
}

\begin{abstract}
The development of security visualization applications must involve the user in the design process in order to create usable systems. However, it is all too easy to lose track of the user during the design and development process, even though upfront investment in extensive user requirements gathering has proven benefits. To address this challenge, we adapt a user-centered design method called personas that enables effective requirements capture for varying scopes of requirements-gathering efforts, and, when used properly, keeps the user involved at every step of the process from design to evaluation.
\end{abstract}

\section{Introduction}

The need for usability in security and visualization interfaces is well-documented in (Adams and Sasse, 1999; Cranor and Garfinkel, 2005; Erbacher et al., 2002; Zurko, 2005). Unfortunately, the failure to achieve usability due to neglect or misunderstanding user requirements is also well-known (Jarzombek, 1999; Standish, 1994, 2001). The domain of human-computer-interaction (HCI) provides a wealth of methods, best practices and approaches for mitigating software failures due to missing user requirements. However, for those unfamiliar with classic user-centered design (UCD) methods, the HCI literature can be daunting and easily misapplied without specialized expertise (Seffah, 2003). Additionally, the methods themselves can be too heavy-weight or resource-intensive to successfully apply to smaller projects, research efforts, or projects with tight deadlines, all of which are common among security visualization applications. As such, there seems to be a gap in the application of effective HCI methods in the computer security domain; specifically

J. Stoll and W.K. Edwards

Georgia Institute of Technology, School of Interactive Computing, Atlanta, Georgia

D. McColgin, M. Gregory, and V. Crow

Pacific Northwest National Laboratory, Richland, Washington 
methodological details about how to ensure that user requirements are indeed reified in the resulting software design.

We address this gap through an in-depth exploration of an HCI methodology aimed at translating user requirements data into the software prototypes. We also demonstrate through a case study how a light-weight user-centered design process can be used to better ensure the usability of the resulting software. The method we discuss, personas, provides a framework for effectively using the user in order to get the user requirements right. This method is a user requirements capturing technique arising out of the Participatory Design philosophy (Grudin and Pruitt, 2002). The value we gained through an adaptation of personas to a security visualization project shows similarity to so-called 'discount usability' methods that can offer cost-effective measures that improve usability even with limited resources (Nielsen, 1995).

In what follows, we first describe the use of personas as a design method and situate this technique in the context of other human-computer interaction (HCI) methods. In Sect. 3, we describe a use case where this method is applied to the requirements gathering phase of a security visualization project. In Sect. 4 we discuss the lessons learned and how this method might be applied for security visualizations more broadly before concluding in Sect. 5 .

\section{Overview of the Personas Method and Related Work}

As with any software application, the first step in building a usable security visualization is to have a good understanding of user requirements. Bowles (2006) suggests the following six steps for the requirements gathering and specification phase of user-centered design approach:

1. Define the product

2. Define the user

3. Define done

4. Define the user interface

5. Define the functional requirements

6. Define the non-functional requirements and constraints.

This and other frameworks help organize the requirements gathering phase of software development but do not necessarily ensure that the user needs actually drive the design of the security visualization tool from start to finish. Anecdotal evidence attests to the ease of overlooking user requirements and designing software for ourselves rather than the user; or designing solely based on technical considerations. We also believe questions concerning design tradeoffs must be determined by the user's needs. We argue that it is not enough to merely define the user, but rather usable software is better achieved through utilizing the user throughout the design and development process; and we believe this can be accomplished for the security visualization projects in part through the use of personas.

In short, a persona is an archetypical user that captures a range of user needs in a coherent narrative (Cooper, 1999). The basic idea behind a persona is that one can 
design for a range of users by designing for a single fictional user called a persona which effectively becomes the representative user by embodying the needs of the selected user population (Cooper, 1999). Where fundamental characteristics are in conflict, multiple personas can be developed to account for diversity in the target users. As such, the personas method provides a way to define, represent, and utilize user requirements (Adlin et al., 2006; Cooper, 1999). It does so by providing a foundation for developing usability metrics, task identification and analysis, and devising a coherent design rationale. Additionally, this method facilitates collaboration among project members and stakeholders since user requirements can be referred to more succinctly via individual personas created for the project.

While there are a number of approaches to defining user requirements (i.e. scenario-based design, storyboards, modeling, and contextual inquiry (Dix et al., 2004; Holtzblatt and Beyer, 1999; Young, 2002)), we argue for the complementary use of personas because this method creates the explicit definition of an ever-present user (Grudin and Pruitt, 2002). Personas should be grounded in a strong understanding of real-world users. Contextual inquiry offers proven methods to gather the raw data, from interviews to longitudinal observation of work practice. Once synthesized into personas, this captured data can be used for varying types of task analysis. We agree with Pruitt and Grudin (2003) that scenario-based design also works well with personas. Scenarios involve descriptions of users performing a task, including the relevant details that might drive system requirements. Scenarios bring to light many technical and functional requirements inherent in accomplishing a task, but tying them to personas can add users' broader goals, social and environmental factors, and relevant information about skills, attitudes, and other factors which need to be considered. These processes working together form a solid foundation for the design of usable systems. Personas can even be used along with other techniques to perform summative evaluation (Dix et al., 2004), as discussed in more detail in Sect. 5.

Viewing potential users as collaborators in the design process and co-creators of the eventual product or prototype is a central thrust behind the participatory design philosophy. The personas method, which grows out of participatory design, makes it more feasible to involve the user in every step of the software development process beginning with the requirements gathering phase. The consequences of unusable security visualizations can be severe (especially if they are for national security or critical infrastructure purposes). Therefore utilizing the personas method which creates an ever-present user seems appropriate, especially as personas has a rich history of successful use by both practitioners and researchers in significantly improving usability across domains (Adlin et al., 2007; Grudin, 2006; Grudin and Pruitt, 2002; Nieters et al., 2007).

\subsection{Personas Method}

Creating a persona consists of identifying and capturing significant details that shape the users' needs. The details considered in a persona include: goals, preferences, challenges and context that influence or dictate what users would need in 
an application. User characteristics are based on data gathered in the "real world". Adlin et al. (2007) provides explicit steps to guide the process of creating a persona. We prefer this five-step method because the persona creation process is explicitly data-driven compared to other more anecdotal approaches (Grudin and Pruitt, 2002). The five steps are:

1. Define the user population and gather data on target users

- Determine the user population.

- Collect user data related to the target population.

- Consider other users such as the international market, disabled persons and the user who is outside the target population.

\section{Transform data gathered into a fictional user or persona}

- Write-up the details into a persona narrative or description.

- Create a foundation document based on the narrative for each persona that is created.

3. Make the persona personable and introduce as "members" of the team

- Create photos and names for each persona.

- Cross-check each persona with "real world" users.

- Introduce each persona to team members and other stakeholders by holding a kick-off meeting.

4. Consult personas in the design decision-making process throughout the project

- Ask how a particular persona may react to particular features or lack thereof.

- Create scenarios using each personas to highlight needed features.

- Create additional documents such as feature-design maps or persona comparison poster.

5. Evaluate design based on persona requirements; evaluate whether to reuse or discard personas

- Use personas to determine the usability of the resulting prototype.

- If choosing to reuse, continue collecting data about personas to revise them as new user data becomes available and continue to further refine and enrich personas by adding new details such as learning style, book usage patterns, etc.

\subsection{Related Work}

The extent to which personas are used and the detail captured and used in the five steps are widely varied. Personas themselves can include elaborate personal details and there is variance in how organizations encourage their adoption within a team. Although the personas method is still relatively new, a number of companies have adopted it successfully, including Cisco, NYT.com, Best Buy, Zylom, Pfaltzgraff, 
and Medco Health (Adlin et al., 2007; Nieters et al., 2007; Spool, 2006). Some of these companies have written about their best practices and yielded guidance on how to best utilize aspects of the personas method. For example, Microsoft markets their persona internally to larger development teams through the use of swag such as mugs with persona photos, mouse pads, posters and playing cards. Similarly, Yahoo! Media threw a party so the team could "meet" the personas (Adlin et al., 2006; Grudin and Pruitt, 2002; Klee and Goodwin, 2001; Spool, 2006).

However, in this investigation, we apply personas to a small research and design project. In doing so, we demonstrate how lighter-weight requirements gathering can be well represented in development settings with the smaller teams, budgets, and shorter timelines that likely characterize most current security visualization design. We provide a use case of personas by introducing a computer security persona and discuss how that persona can be used to increase the usability security visualizations.

\section{Case Study: First Look}

Our implementation of personas was adapted to the research needs of the first look visualization research project driven by a pressing need: As professional information analysts approach a large and dynamic source of data, how can meaningful changes in information be represented and summarized to a user? Analysts spend an inordinate amount of time probing and orienting to their information space before moving on to productive analysis of the relevant portions. Our goal is to develop methods to reduce the time it takes to prioritize work and start analysis. As new data pours in, many aspects change but the significance of these changes depends on type, magnitude, and the perspective of the user.

Our designs need to be domain independent and with such variety in users and data, we needed a way to organize our requirements. We met with a varied set of individuals who are tasked with analyzing content of dynamic, large datasets. From these discussions, it became clear that the use of personas would greatly aid in scoping user requirements. Given the research nature of the project, we could not afford the time or budget to create elaborate personas. Thus, we followed the five steps outlined above for persona building, but made alterations based on our needs and constraints. Our tailored adaptation of personas has been instrumental in guiding our work and is evidence of the robustness of this approach to different scales and domain applications.

\subsection{Five Steps to Persona Implementation}

\subsubsection{Step One: Defining a User Population}

The First Look project as a whole is aimed at supporting basic problems that are common to a broad range of information analysts. Personas helped us organize 
requirements into cogent sets, contextualized by the myriad details common to a particular organization or user type. Creating the personas also brought to light a number of conflicting assumptions that may otherwise have remained hidden. We chose three initial user types: a policy analyst, an intelligence analyst, and a cybersecurity analyst. Gathering data on similarities and unique properties across these three analysis domains continues to inform our design decisions.

Dix et al. (2004) and Young (2002) describe a variety of ways that user data can be collected through both direct means (engaging real-world users) and indirect means (relying on existing data about the target user). We mainly relied on indirect data gathering, borrowing from prior experience with analysts and analyzing many different analyst job postings, organizational charts, and online descriptions. While more exhaustive methods of contextual inquiry have demonstrable benefits, they can often be expensive in time and resources. There can also be problems of user access, client buy-in, and training the project team member. Particularly when resources are limited, personas from other efforts can also be reused or repurposed as we hope ours will be, and personas still have life-cycle benefits even with lower-cost data gathering.

\subsubsection{Step Two: Transform Data into A persona (or Personas)}

The primary outcome of this step is the creation of what is called a foundation document. This document offers a primary means for communicating the persona to all other team members and stakeholders. This method also explicitly links the details of the persona with the supporting user data, i.e. according to Grudin and Pruitt, this document should contain, in narrative form, the details gathered in the first step (2002). They suggest an extensive list of information to include. For our needs, we created the narrative foundation document with the following set of details:

- Job position

- Background which included education and computer skills

- Overall goals

- Work environment which included communication/collaboration activities

- Job duties

- Characteristics of the analyst's particular information space, such as raw data sources and formats, analysis tools, analytical products they created and the target audiences.

The details we gathered are not as extensive as Grudin and Pruitt recommend (2002). We selected them to capture the salient aspects of users for our research efforts. If target security visualization users were, for example, frequently telecommuter working from home then more detailed personas might include details about their home environment and work habits.

The temptation exists to ignore the narrative format and to create a "laundry list" of user requirements based on the user data gathered in the previous step and then to use this as the requirements list. However, there is good evidence that the narrative 
Fig. 1 "Foundation Document" excerpt from the persona Frank

\section{Frank Kreuse}

Cyber Analyst, EOBU (a defense industry supplier)

\section{Background:}

Frank has a B.S. in Information Systems. He specialized in technical writing and has CISSP certification. His training, experience and certification establishes him as an expert computer user.

Work Environment and Information Management: Frank has access to sophisticated data capture tools but better analytic tools are still lacking. Much of the data that he works with is generated by state-of-the-art network scanning tools. However, he primarily relies on Excel to keep track of the data that is of interest to him so that he can perform analyses such as "what if" queries.

Duties:

Part of Frank's duties is to produce risk analysis reports based on test results derived from system assessment tools such as Tivoli Netview and DISA SRR.

form is more effective at keeping the user in the software design and development loop. Psychological evidence demonstrates that a narrative is more engaging and more inspiring than a rote list, boosting motivation in designers, developers, and even clients to use the requirements more effectively (Grudin et al., 2006; Nieters et al., 2007). The persona also becomes an efficacious and convenient way to quickly convey a set of requirements among the project team or with other groups. The partial document in Fig. 1 is part of foundation document for one of our personas, "Frank, the Cyber Analyst".

Since the creation of personas enabled us to have a systematic method for representing user groups, we gained richer understanding of analysts that came from making the requirements explicit. We became more cognizant of the significant differences among different analyst types such as information assurance analysts vs. policy analysts. The bounds of our problem space were defined by the multiple personas we developed to account for the breadth of user types we needed to support; and these insights would not have been as easily or naturally gained with non-narrative, non-personal lists of user features.

\subsubsection{Step Three: Personalize the Persona(s)}

The personas become more real to the team members the more they know about them. Grudin and Pruitt (2002) argues that photos, especially candid, more realistic photos (from freely available stock) lend to the "personability" of the personas. The combination of name and picture helps the team to remember and refer to them. The personas we created to match our three user types were each given a name and an associated photo. They were introduced to the First Look team by sharing the persona foundation documents at team meetings. Everyone on the team then had the opportunity to review the personas and provide feedback. As the project continued with technical discussions and research, we (almost naturally) began to refer to 
Table 1 Three personas created for the First Look project

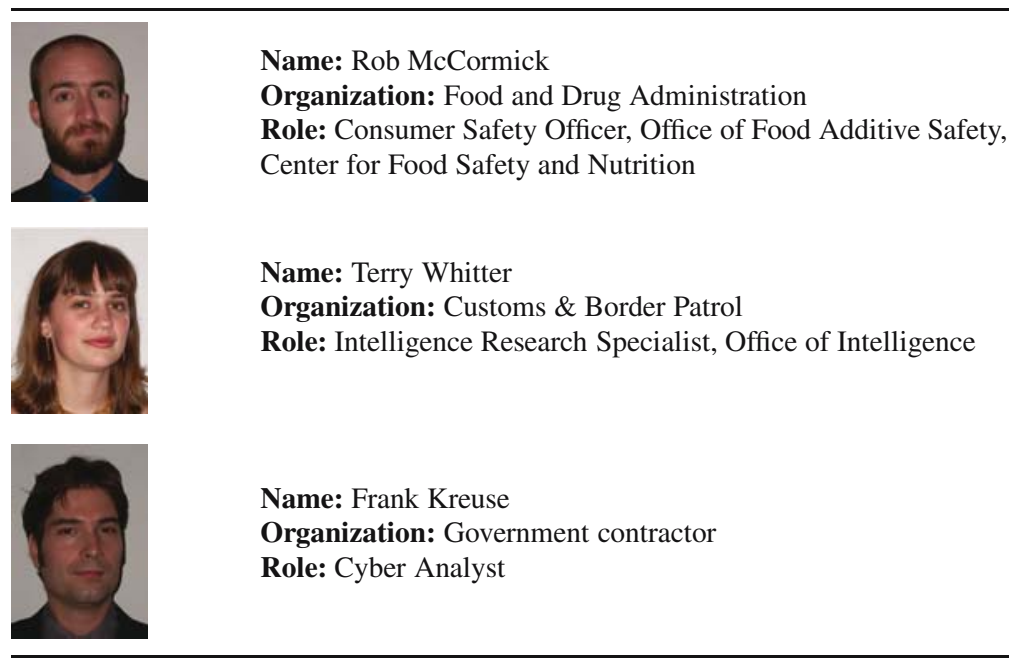

personas by name as we discussed aspects that would affect them to reinforce their utility after their introduction and make clear the role they would play as team members. Our three personas were "Frank, the Cyber Analyst", "Terry, the Intelligence Researcher", "Rob, the Consumer Safety Officer", represented below in Table 1.

\subsubsection{Step Four: Consulting Personas in the Design Decision-Making Process}

Once personas are created, they can be "consulted" with when design decisions arise particularly during the development phase. In order to facilitate this process, two matrices can be created: (1) a data-to-features matrix and (2) a persona-weighted feature matrix. To generate these matrices, we created multiple scenarios for our personas to explore requirements. These scenarios stemmed from specific tasks and situations where the persona analyst must deal with tracking information change in a large information space. For example, Frank, as a cyber analyst, was run through a scenario where he had to validate the emergence of a threat pattern using disparate data sources. How Frank performed in the scenario was constrained by the persona characteristics that he was given, i.e. his job description, the resources that he had available and time constraints.

These scenarios, tightly coupled with the personas, allowed us ultimately to create a weighted "feature matrix" (sample show in Fig. 2) that let us explore the importance of individual system features relative to the other features based on persona needs. This matrix was then used to explore initial design directions and for creating a design map that will be used to inform our prototype design.

The data and the features inferred from the persona and scenario data are tabulated and used to inform the persona-weighted feature matrix. Figure 3 is an example of how these requirements for each personal can be weighted to aid in decision 


\begin{tabular}{|c|c|c|c|}
\hline \multicolumn{3}{|c|}{ User Data } & Features \\
\hline $\begin{array}{l}\text { Frank } \\
\text { Cyber Analyst }\end{array}$ & $\begin{array}{l}\text { Terry } \\
\text { Intelligence Researcher }\end{array}$ & $\begin{array}{l}\text { Rob } \\
\text { Consumer Safetry Officer }\end{array}$ & \\
\hline $\begin{array}{l}\text { "Frank is not always } \\
\text { the person required } \\
\text { to respond to intrusions. } \\
\text {...he must be aware of } \\
\text { and be able to access } \\
\text { information about how } \\
\text { each incidenct is being } \\
\text { handled by the team. } \\
\text {...network monitoring } \\
\text { information comes } \\
\text { from a variety of tools so } \\
\text { Frank needs to know } \\
\text { where the information } \\
\text { came from." }\end{array}$ & $\begin{array}{l}\text { "Terry must perform her } \\
\text { analysis in a highly } \\
\text { collaborative environment } \\
\text { and the data she receives } \\
\text { from the Intelligence } \\
\text { support staff comes from } \\
\text { many varied sources. } \\
\text {... at times she must do } \\
\text { quality control on the } \\
\text { information she receives } \\
\text { from the newer support } \\
\text { staff by making sure they } \\
\text { are coming from credible } \\
\text { sources." }\end{array}$ & $\begin{array}{l}\text { "Rob works for an agency } \\
\text { that utilizes antiquated } \\
\text { computer and information } \\
\text { systems. } \\
\text { One of the daily challenges } \\
\text { that Rob faces is keeping } \\
\text { track of the many alerts } \\
\text { he receives about food } \\
\text { safety incidents to be } \\
\text { investigated. He relies on } \\
\text { where the alert came from } \\
\text { to determine priority for } \\
\text { investigation." }\end{array}$ & $\begin{array}{l}\text { 1. Functional: } \\
\text { Need a } \\
\text { summary of } \\
\text { the metadata } \\
\text { about the } \\
\text { information } \\
\text { being used in } \\
\text { analysis. }\end{array}$ \\
\hline
\end{tabular}

Fig. 2 "Data-to-features" matrix excerpt

\begin{tabular}{|l|c|c|c|c|}
\hline \multicolumn{1}{|c|}{ Features } & \multicolumn{3}{|c|}{ Persona Weights } \\
\hline & $\begin{array}{l}\text { Frank } \\
\text { (weight = 40) }\end{array}$ & $\begin{array}{l}\text { Terry } \\
\text { (weight = 40) }\end{array}$ & $\begin{array}{l}\text { Rob } \\
\text { (weight = 20) }\end{array}$ & $\begin{array}{l}\text { Weighted } \\
\text { Priority }\end{array}$ \\
\hline $\begin{array}{l}\text { 1. Functional: } \\
\text { Need a summary of the } \\
\text { metadata about the } \\
\text { information being used in } \\
\text { analysis. }\end{array}$ & $\mathbf{2}$ & $\mathbf{2}$ & $\mathbf{2 0 0}$ \\
\hline $\begin{array}{l}\text { 2. Non-functional: } \\
\text { Need information change } \\
\text { alerts to be rapidly } \\
\text { customize-able. }\end{array}$ & $\mathbf{2}$ & $\mathbf{1}$ & $\mathbf{0}$ \\
\hline \\
$\begin{array}{l}\text { weight }=\text { percentages totaling to 100\% or on a scale such as 1-5 } \\
\text { score }=-1 \text { harms persona } \\
0 \text { does not matter to persona if the feature is there or not } \\
+1 \text { helpful to the persona } \\
+2 \text { is a must-have feature for the persona }\end{array}$ \\
\hline
\end{tabular}

Fig. 3 Persona-weighted feature matrix excerpt

making in the design process (Adlin et al., 2007). For each of the features identified in the data-to-feature matrix, an inference is made by the designer or development team to determine whether such a feature is helpful to the persona, harmful or neither. The personas themselves are also assigned a weight according to the priority of the visualization project. For project First Look, our focus was on designing primarily for the cyber analyst, Frank rather than the policy analyst, Rob. Therefore, Frank was assigned a heavier weighting than Rob. Consequently, the project development process will focus primarily on features which Frank finds important. Also, when design conflicts arise between catering towards Rob's need vs. Frank's, the assigned weighting should provide direction as to which tradeoffs to make in the design.

Our choice to use a feature matrix is partly based on Meuller's (Adlin et al., 2007) recommendations for deciding which attributes, based on which users, are crucial for inclusion in the design. Also, both matrices can be combined and used as a handy reference card throughout the development process, similar to the one created and used at Yahoo! Media with success. Designers and developers were able to refer to the card as they were in the process of actually coding the prototype. 
This is particularly important as the experience of software engineering tells us that critical design decisions often occur later on realities of development unfold (Dix et al., 2004). Again this points to the utility of the personas method to enable the user to be ever-present throughout the duration of the project.

By comparing the personas we were able to identify which features would need to vary across the range of personas and which were held in common. This informed important decisions about system architecture. In fact, we concluded that a standalone software tool would actually hinder our users by forcing them to change their workflow and have yet another tool to learn when our goal is to provide rapid, usable orientation. Thus, we explored options such as creating extensions to existing tools and providing services that could be combined into custom applications. The varying time constraints and use environments of the diverse personas led us to explore multimodal interface options and various blends of push and pull technologies, as illustrated by Fig. 4. (It should be noted, however, that the task of generating actual designs from the functional and non-functional requirements identified is often more dependent on the designer's inspiration than other factors.)

Personas helped to reveal some natural connections between technical resources and user needs through functional and non-functional requirements. At other times, one or more of the project members asserted opinions about certain features that needed to be included while others dissented. In these situations, discussing the feature in light of the persona's need helped to bring project members to an agreement regarding the design decision. Assuming the role of a persona can illuminate the problem in a new light. When no personas seem applicable it may highlight the need for additional unforeseen requirements and an opportunity to further define the problem space.

Using these three matrices to inform design decisions throughout the development lifecycle can provide a means to coherently connect design decisions made with user requirements data.

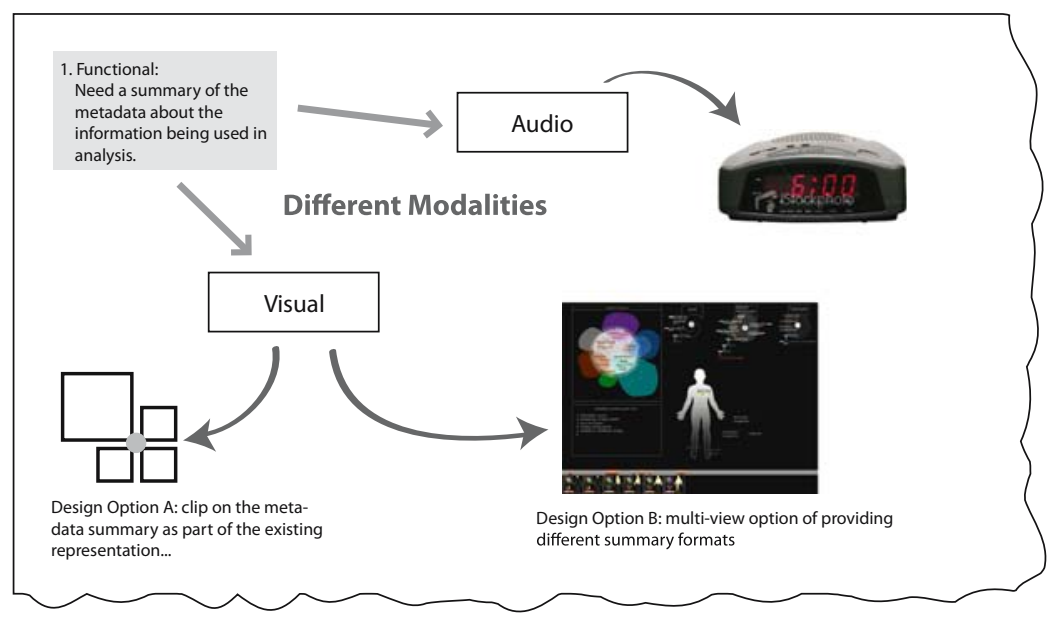

Fig. 4 "Feature-to-design" map excerpt 


\subsubsection{Step Five: Evaluate Results and Persona Retention}

In this step, the personas are used to evaluate the resulting system. In the First Look project we have still been in the design/prototyping stage and have not yet used the personas for this last step of evaluation. However, we intend to use the weighted feature matrix and design map for this purpose. For our research-oriented project, we also plan on using personas as a perspective in cognitive walkthroughs, a baseline in heuristic evaluations, and possibly as guidelines for prioritizing quality assurance. The literature provides numerous examples of the successful use of personas for evaluating the software in a variety of ways (Adlin et al., 2007).

We plan to retain the personas foundation document and other related documents to make them available as part of a library for other projects with similar goals and user populations. Projects with no resources for requirements gathering can benefit from the rich representation of users and requirements, or they may provide a starting point for bootstrapping efforts on related problems.

\subsection{Discussion}

We have found that in this small research project the use of personas was crucial to design processes, and continues to be useful throughout the implementation. One challenge we encountered that has been experienced by others (Adlin et al., 2006; Holtzblatt and Beyer, 1999; Klee and Goodwin, 2001; Spool, 2006) is gaining acceptance of personas as additional members of the team. It is not surprising that real-world team members need time to learn how to work with their persona colleagues. For our smaller core team, the foundation documents and consistent invocation were sufficient. As we offer the personas as resources to more people and other projects we may investigate other options, such as Yahoo! Media's persona party, though this approach seems more appropriate for much larger teams who are already facing cohesion challenges. However, as it did for the First Look project, personas can serve as a common rallying point for project engagement which may have been otherwise lacking.

\section{Application to Security Visualizations}

In the case study we presented here, personas were useful for logically and coherently grouping requirements, designing with real user needs in mind, and giving all team members a shared understanding of project goals and priorities through a memorable narrative. The personas method is appropriate for capturing and utilizing user requirements particularly for security visualization system design vis-à-vis other security problems. Unlike other security problems such as sensor data capture or anomaly detection, a security visualization system must be designed with the 
human user in mind because analysts will be interacting directly with the interface to help with their tasks. Thus, making sure the user needs are appropriately addressed is particularly critical and the personas method helps to ensure that this is the case throughout the duration of the security visualization project.

Though our specific research project was not geared solely to computer security alone, Frank, our cyber analyst persona, gave us insight into the processes in common across analysis domains as well as the unique properties of the cyber analyst. For example, like other profession information analysts, Frank required tools to help manage the information being gathered to support his hypothesis of threat pattern development. However, unlike other analysts, Frank did not have to cope with new information arriving at irregular intervals. He did not have a need for indicator level alerts to prompt him when new information had arrived, a feature required by both the intelligence and policy analysts.

Also, our project goals led us focus on how Frank coordinates with other staff and integrates information from email, alerts, and social connections with changing data about current system performance to protect the network. We acknowledge that for many applications within security visualization, there are multiple user types and a project with a different focus may capture different requirements in a given persona. Having a suite of personas to represent the range of functionality needed may help security visualization designers better understand and articulate the degree of flexibility needed for each functional/non-functional requirements identified.

Projects specifically targeting cyber-security may need to create sub-type personas to more precisely cover the range of intended users such as system administrators, cyber or information assurance analysts, and network administrators, among others. Variations within the user types can be significant enough to warrant finergrained representations of the user population that can inform design (Conti et al., 2005; D'Amico and Kocka, 2005; Goodall and Lutters, 2004; Yurcik et al., 2003). The use of sub-types of users could be exploited in the same way that we exploited our three user types: each of them can help define features, and give a weighting to those features to inform and direct user-centered design alternatives.

Relevant details could also vary. Applications interested in remote system monitoring might embed in personas information about the home environment or experience and skill with mobile devices. A visually rich application supporting interaction with massive data might also have a persona that, among other things, represents the substantial color-blind user population.

As a developing field, security visualization encompasses a broad range of research efforts, prototypes, and commercial products. With so much at stake, we must ensure that these systems are usable and target the right problems by effectively involving users. Security visualization applications are highly varied, and while the literature provides examples of effective persona use for large-scale software projects, our case study demonstrates that they can be adapted to smaller scale projects, including research, to effectively be centered on the user in the process. 


\section{Conclusion}

Security visualization applications intended to help users deal with massive data must be more than just aesthetically pleasing or purely functional. They must be share the users' priorities and be usable or their benefits are compromised. Once requirements are gathered, personas offer an adaptable way to keep the user involved in all of the design, development, and evaluation that will affect usability and adoption of the application. The accessibility of the personas method allows all team members from developers to quality assurance testers to utilize the personas, and thereby sharing a common understanding of the goals and priorities. They can enable even collaborators, clients, and other projects with similar needs to easily share this improved understanding.

Our experience also demonstrates the adaptability of the persona method to research and efforts that cannot prioritize expensive requirements-gathering approaches. We do not contend that personas is the sole method for achieving usercentered design, but rather that they particularly enable the user to remain in the process as a focal point throughout the life-cycle of the project. In other words, they help to ensure we are solving problems that are important to the users in truly usable ways.

\section{References}

Adams A, Sasse MA (1999) Users are not the enemy. Communications of the ACM 42, 12, pages 40-46. doi: 10.1145/322796.322806

Adlin T, McGrane K, Pruitt J, Rosenstein A, Goodwin K, Muller M, Hynes C (2006) Panel: Putting Personas to Work. CHI '06 Proceedings

Adlin T, Pruitt J, Grudin J (2007) The Persona Lifecycle: What Personas Are, Why They Work, and How to Create and Use Them. CHI '07 Course Notes, San Jose, CA

Bowles JB (2006) Better software reliability by getting the requirements right. Reliability and Maintainability Symposium. doi: 10.1109/RAMS.2006.1677359

Conti G, Ahamad M, Norback R (2005) Filtering, fusion and dynamic information presentation: towards a general information firewall. IEEE International Conference on Intelligence and Security Informatics

Cooper A (1999) The Inmates are Running the Asylum: Why High Tech Products Drive Us Cranor L, Garfinkel S (2005) Security and Usability: Designing Secure Systems that People Can Use

Crazy and How to Restore the Sanity. Macmillan

D'Amico A, Kocka M (2005) Information assurance visualizations for specific stages of situational awareness and intended uses: lessons learned. Proceedings of the IEEE Workshops on Visualization for Computer Security. doi: 10.1109/VIZSEC.2005.13

Dix A, Finlay J, Abowd G, Beale R (2004) Human-Computer Interaction, 3rd Ed. London: Prentice-Hall

Erbacher RF, Walker KL, Frincke DA (2002) Intrusion and misuse detection in large-scale systems. IEEE Computer Graphics and Applications 22, 1

Goodall J, Lutters W (2004) The work of intrusion detection: rethinking the role of security analysts. 10th Americas Conference on Information Systems

Grudin J (2006) Why personas work: The psychological evidence. In Pruitt J, Adlin T (eds). The Persona Lifecycle. Amsterdam: Elsevier 
Grudin J, Pruitt J (2002) Personas, participatory design and product development: an infrastructure for engagement. The Participatory Design Conference '02

Grudin J, Pruitt J, Adlin T (2006) The Persona Lifecycle: Keeping People in Mind throughout Product Design. Los Altos, CA: Morgan Kaufmann. http://research.microsoft.com/ jgrudin/ publications/personas/PersonasChapter.pdf

Holtzblatt K, Beyer H (1999) Contextual design: using customer work models to drive systems design. Extended Abstracts on Human Factors in Computing Systems. doi:10.1145/ 632716.632801

Jarzombek, SJ (1999) The 5th Annual Joint Aerospace Weapons Systems Support, Sensors, and Simulation Symposium (JAWS S3), Proceedings

Klee M, Goodwin K (2001) Personas and Goal-Directed Design: An Interview with Kim Goodwin. http://www.uie.com/articles/goodwin_interview/. Accessed on 10 September 2007

Nielsen J (1995) Scenario-based design: envisioning work and technology in system development book contents: Scenarios in discount usability engineering, pages 59-83. New York, NY: Wiley

Nieters J, Ivaturi S, Ahmed I (2007) Making personas memorable. In CHI '07 Extended Abstracts on Human Factors in Computing Systems. doi: 10.1145/1240866.1240905

Pruitt J, Grudin J (2003) Personas: Practice and Theory. Conference on Designing For User Experiences '03. doi: 10.1145/997078.997089

Seffah A (2003) Learning the Ropes: Human-centered Design Skills and Patterns for Software Engineers' Education. ACM Interactions '03

Spool J (2006) Yahoo's Approach to Keeping Personas Alive. http://www.uie.com/brainsparks/ 2006/05/18/yahoos-approach-to-keeping-personas-alive/. Accessed on 10 September 2007

The Standish Group International Inc. (1994) The CHAOS Report

The Standish Group International Inc. (2001) The CHAOS Report

Young R (2002) Recommended Requirements Gathering Practices. CrossTalk: The Journal of Defense Software Engineering. http://www.stsc.hill.af.mil/crosstalk/2002/04/young.html. Accessed on 10 September 2007

Yurcik W, Barlow J, Rosendale J (2003) Maintaining perspective on who is the enemy in the security systems administration of computer networks. ACM CHI Workshop on System Administrators Are Users, Too '03

Zurko M (2005) User-centered security: stepping up to the grand challenge. Computer Security Applications Conference (ACSAC) '05 